\section{(2) OPEN ACCESS}

\title{
CSF and blood Kallikrein-8: a promising early biomarker for Alzheimer's disease
}

\author{
Sarah Teuber-Hanselmann, ${ }^{1}$ Jan Rekowski, ${ }^{2}$ Jonathan Vogelgsang, ${ }^{3}$ \\ Christine von Arnim, ${ }^{4,5}$ Kathrin Reetz (1) , ${ }^{6}$ Andreas Stang, ${ }^{7}$ Karl-Heinz Jöckel, ${ }^{2}$ \\ Jens Wiltfang, ${ }^{3,8}$ Herrmann Esselmann, ${ }^{3}$ Markus Otto (D) , ${ }^{4}$ Hayrettin Tumani, ${ }^{4}$ \\ Arne Herring, ${ }^{1}$ Kathy Keyvani (D) ${ }^{1}$
}

\begin{abstract}
- Additional material is published online only. To view please visit the journal online (http://dx.doi.org/10.1136/ jnnp-2019-321073)
\end{abstract}

For numbered affiliations see end of article.

\section{Correspondence to} Professor Kathy Keyvani, Institute of Neuropathology, Faculty of Medicine, University of Duisburg-Essen, Essen 45147, Germany; kathy. keyvani@uk-essen.de

$\mathrm{AH}$ and $\mathrm{KK}$ contributed equally.

Received 24 April 2019 Revised 15 July 2019

Accepted 23 July 2019 Published Online First 1 August 2019

Check for updates

(C) Author(s) (or their employer(s)) 2020. Re-use permitted under CC BY-NC. No commercial re-use. See rights and permissions. Published by BMJ.

To cite: Teuber-Hanselmann S, Rekowski J, Vogelgsang J, et al. J Neurol Neurosurg Psychiatry 2020:91:40-48.

\section{ABSTRACT}

Objective There is still an urgent need for supportive minimally invasive and cost-effective biomarkers for early diagnosis of Alzheimer's disease (AD). Previous work in our lab has identified Kallikrein-8 (KLK8) as a potential candidate since it shows an excessive increase in human brain in preclinical disease stages. The aim of this study was to evaluate the diagnostic performance of cerebrospinal fluid (CSF) and blood KLK8 for AD and mild cognitive impairment (MCl) due to $A D$.

Methods In this multi-centre trans-sectional study, clinical and laboratory data as well as CSF and/or blood serum samples of 237 participants, including 98 patients with mild $A D, 21$ with $M C l$ due to $A D$ and 118 controls were collected. CSF and/or serum KLK8 levels were analysed by ELISA. The diagnostic accuracy of KLK8 in CSF and blood was determined using receiver operating characteristic (ROC) analyses and compared with that of CSF core biomarkers A 342 , P-tau and T-tau.

Results The diagnostic accuracy of CSF KLK8 was as good as that of core CSF biomarkers for AD (area under the curve $(A \cup C)=0.89)$ and in case of $\mathrm{MCl}(\mathrm{AUC}=0.97)$ even superior to CSF A 342 . Blood KLK8 was a similarly strong discriminator for $\mathrm{MCl}(\mathrm{AUC}=0.94)$ but slightly weaker for $\mathrm{AD}(\mathrm{AUC}=0.83)$.

Conclusions This is the first study to demonstrate the potential clinical utility of blood and CSF KLK8 as a biomarker for incipient $A D$. Future prospective validation studies are warranted.

\section{INTRODUCTION}

There is clear evidence that Alzheimer's disease (AD) starts to damage the brain decades before the first symptoms appear. ${ }^{12}$ This has led to the current consensus that any disease-modifying treatment can be efficacious only when initiated at the earliest stages of the disease. ${ }^{13}$ Despite many efforts during the past 30 years, only three CSF biomarkers for the diagnosis of $\mathrm{AD}$ (ie, $\mathrm{A} \beta_{42}$, total tau and phosphorylated tau) have been hitherto established for clinical practice. ${ }^{4}$ Meanwhile, CSF $\mathrm{A} \beta_{42}$ has been established as a surrogate marker for cerebral amyloid burden assessed by positron emission tomography (PET) scan. Although $A \beta_{42}$ together with total and phosphorylated tau show high accuracy for the differentiation between $\mathrm{AD}$-affected patients and healthy controls ${ }^{5}$ their discriminative power between $\mathrm{AD}$ and other dementias remains insufficient ${ }^{67}$ and although being pathologically altered years before disease onset, ${ }^{1} 89$ their diagnostic value in detecting mild cognitive impaired (MCI) patients due to $\mathrm{AD}$ remains controversial. ${ }^{40-13}$ Furthermore, there is an urgent need for less costly and intrusive, and more widely available blood-based biomarkers that can be used at primary care settings. ${ }^{14}$ While the diagnostic performance of blood-based biomarker tau has been shown to be poor ${ }^{15}$ recent data on the blood-based $A \beta$ measurements by immunoprecipitation coupled with mass spectrometry ${ }^{16}$ demonstrate a high performance in predicting cerebral $\mathrm{A} \beta$ burden. However, the clinical utility and feasibility of this method for reliable detection of $\mathrm{AD}$ patients, in particular at early disease stages and for efficient population screening remain to be verified. As a consequence, new fluid biomarkers are essential if they increase diagnostic precision in combination with the established core biomarkers.

Previous work in our lab has identified Kallikrein-8 (KLK8, alias: neuropsin) as a potential upstream prime mover in the pathogenesis of AD. ${ }^{17}$ We could detect surplus KLK8 mRNA and protein levels in both $\mathrm{AD}$-vulnerable (neocortex and hippocampus) and non-vulnerable (cerebellum) brain areas very early in the course of the disease, that is, in transgenic mice prior to $\mathrm{A} \beta$ pathology onset and in $\mathrm{AD}$ patients in preclinical stages (CERAD A, Braak I-II). Moreover, short-term KLK8 inhibition in moderate stage disease mitigated multiple features of Alzheimer's pathology in transgenic mice. ${ }^{17}$ These results raise the possibility that KLK8 could function as an antecedent biomarker for and a new therapeutic target against AD. ${ }^{17}$ In this unprecedented study we sought to evaluate the diagnostic performance of KLK8 in CSF and blood of $\mathrm{MCI}$ due to $\mathrm{AD}$ and (incipient) $\mathrm{AD}$ patients, also in comparison with the CSF performance of the established core biomarkers.

\section{METHODS}

\section{Study population}

This multicentre cross-sectional study contained 237 participants in total, including 98 patients with (early stages of) $\mathrm{AD}, 21$ patients with $\mathrm{MCI}$ due to $\mathrm{AD}$ (hereinafter referred to as $\mathrm{MCI}$ ) and 118 controls (CON). Three different clinical departments in Germany, namely the Department of Psychiatry 


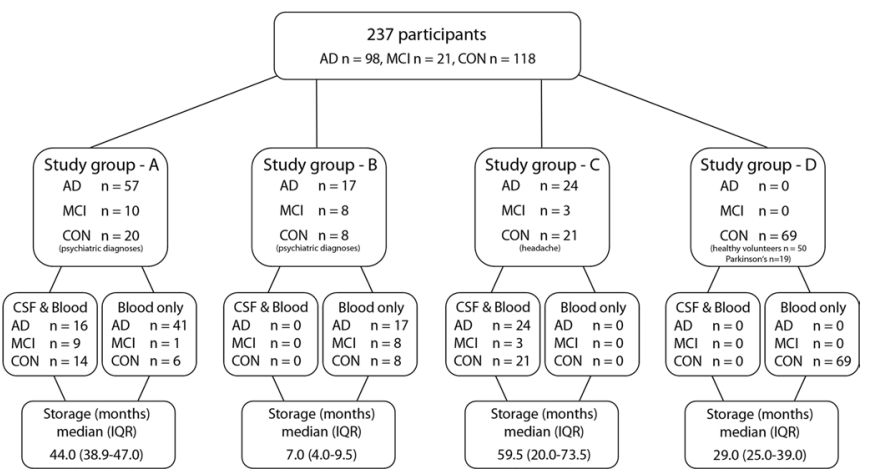

Figure 1 Study population and sample composition. AD, Alzheimer's disease; CON, controls; CSF, cerebrospinal fluid; IQR, interquartile range; KLK8, Kallikrein-8; $\mathrm{MCl}$, mild cognitive impairment due to AD Parkinson's, Parkinson's disease.

and Psychotherapy, University Medical Center Goettingen, the Department of Neurology, University Hospital of Ulm, and the Department of Neurology, RWTH Aachen University took part in the study. Study groups A and B were from Goettingen and consisted of $57 \mathrm{AD}, 10 \mathrm{MCI}$ and 20 controls or $17 \mathrm{AD}$, eight MCI and eight control participants, respectively. Study group C was from Ulm and comprised 24 AD, 3 MCI, 21 control individuals. Study group D came from Aachen and consisted of 69 control participants (for an outline see figure 1).

\section{Approvals and patient consents}

All samples were taken from biobanks of fluid biomaterial at each department. Written informed consent was obtained from all participants at each department before admission to the biobanks.

\section{Clinical diagnoses}

The clinical diagnosis of $\mathrm{AD}$ or $\mathrm{MCI}$ due to $\mathrm{AD}$ was made according to the National Institute on Aging and Alzheimer's Association (NIAAA) criteria ${ }^{18} 19$ based on cognitive assessments in a neuropsychological test battery, including Mini Mental State Examination (MMSE) and CSF testing for the core biomarkers $\mathrm{A} \beta_{42}$, total tau (T-tau) and phospho-tau-181 (P-tau). All AD patients were diagnosed as 'probable $\mathrm{AD}$ dementia with evidence of $\mathrm{AD}$ pathophysiological process ${ }^{, 19}$ and all MCI patients had a 'high likelihood' to convert to $\mathrm{AD}$ according to NIA-AA criteria ${ }^{18}$ (ie, core clinical criteria for $\mathrm{MCI}+\mathrm{de}-$ creased $\mathrm{A} \beta$ and increased P-tau and/or T-tau levels in CSF). Control group consisted of a heterogeneous population. Control patients of the study groups $\mathrm{A}$ and $\mathrm{B}(\mathrm{n}=28)$ received the psychiatric diagnoses affective disorders $(n=24)$, neurotic or stress related disorders $(n=1)$, or schizophrenia $(n=3)$. Control patients of the study group $C$ $(n=21)$ were referred to the outpatient Clinic in Ulm due to tensiontype headache. Control participants of study group D consisted of either healthy voluntary employees at the RWTH Aachen University $(n=50)$ or patients who were diagnosed in the outpatient clinic in Aachen for Parkinson's disease (PD) without dementia (according to Montreal Cognitive Assessment test) $(n=19)$. All clinical diagnoses were first time diagnoses (no AD-related premedication) and were made by experienced physicians at specialised centres at university hospitals. Based on clinical, basic chemical and imaging examinations none of the participants of this study revealed any neoplastic, chronic inflammatory, neurological or noteworthy internal diseases apart from the named above.

Table 1 Demographic, genetic and biochemical data

\begin{tabular}{|c|c|c|c|c|c|c|c|}
\hline & & & & CON & & & \\
\hline & & $A D$ & $\mathrm{MCl}$ & Healthy & Headache & Psychiatric & PD \\
\hline Demographics & & & & & & & \\
\hline Patients & No. & 98 & 21 & 50 & 21 & 28 & 19 \\
\hline Female & No. & 59 & 6 & 21 & 11 & 15 & 10 \\
\hline Male & No. & 39 & 15 & 29 & 10 & 13 & 9 \\
\hline Age (years) & Median (IQR) & $72(64-77)$ & 72 (66 to 77 ) & $66(59-70)$ & $58(51-64)$ & $59(46-64)$ & $68(62-73)$ \\
\hline MMSE score & Median (IQR) & $23(19-25)$ & 28 (27 to 29 ) & NA & NA & $28(27-29)$ & NA \\
\hline MoCA score & Median (IQR) & NA & NA & $30(28-30)$ & NA & NA & $29(27-29)$ \\
\hline Genetic & & & & & & & \\
\hline APOE $\varepsilon 4-I-$ & No. & 12 & 4 & NA & NA & 6 & NA \\
\hline APOE $\varepsilon 4+1-$ & No. & 19 & 4 & NA & NA & 2 & NA \\
\hline APOE $\varepsilon 4+/+$ & No. & 10 & 1 & NA & NA & NA & NA \\
\hline Blood and CSF samples & No. & 40 & 12 & NA & 21 & 14 & NA \\
\hline Blood samples only & No. & 58 & 9 & 50 & NA & 14 & 19 \\
\hline Biochemical & & & & & & & \\
\hline $\mathrm{A} \beta_{42}(\mathrm{pg} / \mathrm{mL})$ & Median (IQR) & $504(424-652)$ & 745 (489 to 1191) & NA & 1034 (917-1225) & 1093 (823-1248) & NA \\
\hline T-tau $(\mathrm{pg} / \mathrm{mL})$ & Median (IQR) & 627 (409-920) & 382 (243 to 683 ) & NA & $215(171-328)$ & 174 (117-272) & NA \\
\hline P-tau (pg/mL) & Median (IQR) & $89(67-117)$ & 56 (43 to 83$)$ & NA & NA & $39(29-45)$ & NA \\
\hline $\mathrm{A} \beta_{42 /} / \mathrm{A} \beta_{40}$-ratio & Median (IQR) & $0.5(0.4-0.7)$ & $0.7(0.5$ to 1.0$)$ & NA & NA & $1.0(0.8-1.2)$ & NA \\
\hline Albumin CSF/blood ratio & Median (IQR) & $5.9(4.3-7.2)$ & 6.6 (5.3 to 9.0$)$ & NA & $6.1(5.0-6.9)$ & $7.1(5.2-9.3)$ & NA \\
\hline
\end{tabular}

Demographic, genetic and biochemical data are shown for every diagnostic group. Control patients are further subdivided into healthy volunteers, patients with tension-type headaches, patients with psychiatric diagnoses, namely affective disorders $(n=24)$, neurotic or stress related disorders $(n=1)$, or schizophrenia $(n=3)$, or patients with Parkinson's disease (PD).

$A D$, Alzheimer's disease; $A \beta_{42 \text { or } 40^{\prime}} \beta$-amyloid 42 or 40 ; CON, controls; $C S F$, cerebrospinal fluid; Headache, patients with tension-type headache; Healthy, healthy volunteers; IQR interquartile range; $\mathrm{MCl}$, mild cognitive impairment due to $\mathrm{AD}$; $\mathrm{MMSE}$, mini mental state examination; MoCA, Montreal Cognitive Assessment; NA, not available; $\mathrm{PD}$, patients with Parkinson's disease; Psychiatric, patients with psychiatric diagnoses; P-tau, phosphorylated tau; T-tau, total tau. 
AD vs. $\mathrm{MCl}$ vs. CON
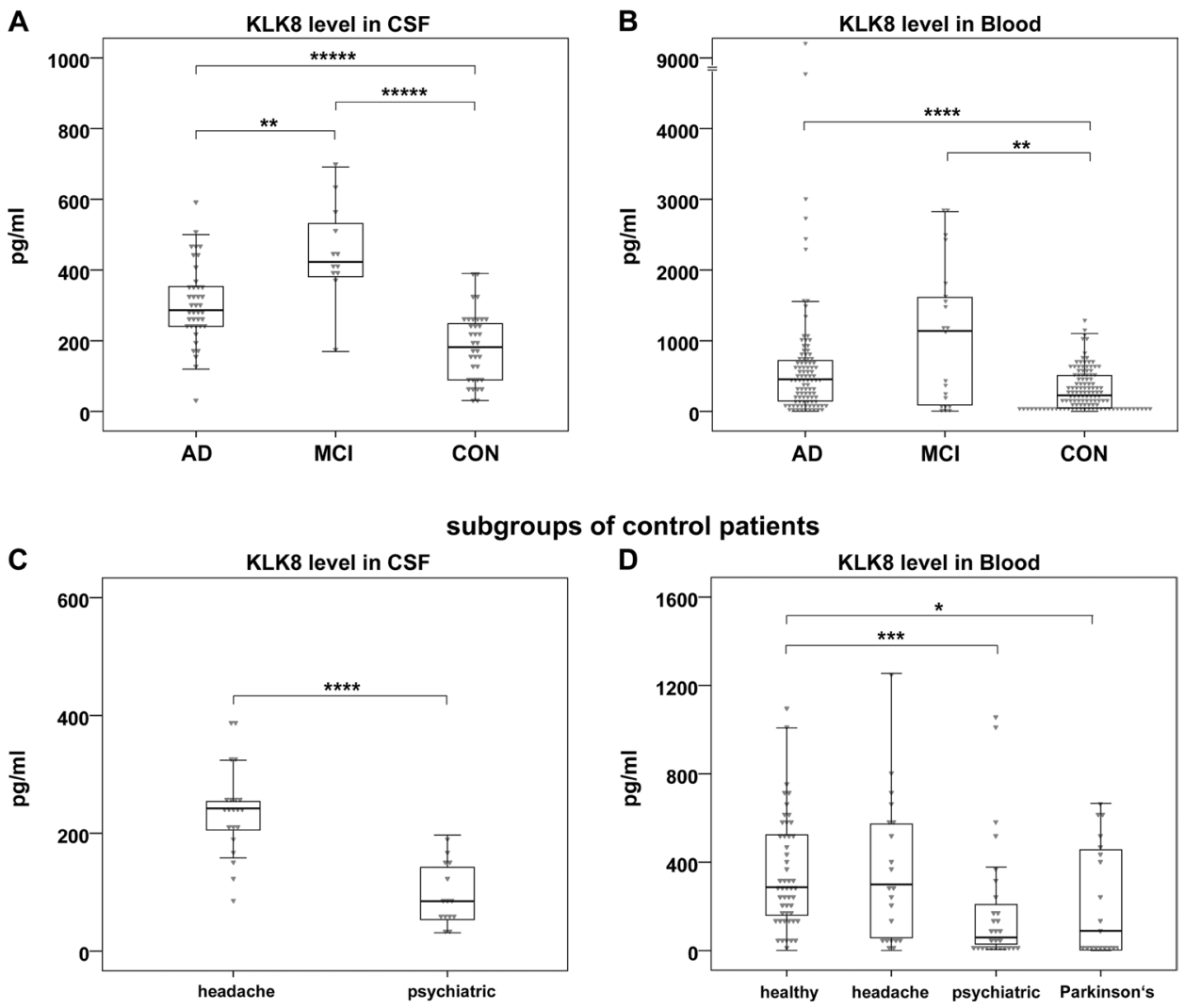

Figure 2 CSF and blood KLK8 levels in different diagnostic groups A, B: comparisons of CSF (A) and blood (B) KLK8 levels between patients with AD ( $n=40$ and $n=98$ for CSF and blood, respectively) or $\mathrm{MCl}(\mathrm{n}=12$ and $n=21$ for CSF and blood, respectively) and controls ( $n=35$ and $n=118$ for CSF and blood, respectively). The two outliers depicted in panel B (KLK8 values: 4775 and $9294 \mathrm{pg} / \mathrm{mL}$ ) were within the normal range of their diagnostic group regarding MMSE, biochemical or genetic data. C, D: comparisons of CSF (C) and blood (D) KLK8 levels between subgroups of control patients. The heterogeneous control group consisted of patients with headache ( $n=21$ for CSF and blood) (C, D) and patients with psychiatric diseases ( $n=14$ and $n=28$ for CSF and blood, respectively) (C, D) as well as healthy volunteers $(n=50)$ and patients with PD without signs of dementia $(n=19)(D)$. Box plots indicate first to third quartile; lines represent medians; whiskers represent $1.5{ }^{*}$ IQR. P values are determined by Wilcoxon test. ${ }^{*} p=0.03 ;{ }^{* *} p \leq 0.003 ;{ }^{* * *} p=0.0001$; ${ }^{* * * *} p \leq 0.00003 ;{ }^{* * * *} p \leq 0.000009$. AD, Alzheimer's disease; CON, controls; CSF, cerebrospinal fluid; Headache, patients with tension-type headache; Healthy, healthy volunteers; KLK8, Kallikrein-8; MCl, mild cognitive impairment due to AD; Parkinson's, patients with Parkinson's disease; Psychiatric, patients with psychiatric diagnoses.

\section{Statistical analyses}

Data are presented as median and IQR. Median KLK8 differences between diagnostic groups are determined by the HodgesLehmann estimator (see online supplementary table S2). None of the outliers were excluded from any of the following analyses, but even after their exclusion the differences remained highly significant. Data distribution was evaluated graphically using histograms and Q-Q-plots. Since at least blood KLK8 did not show normal distribution, the Wilcoxon test was applied for both CSF and blood KLK8 to test the pairwise differences between the diagnostic groups. Additionally, mean \pm SEM, median, Q1, Q3, IQR, skewness, kurtosis, minimum/maximum CSF and blood KLK8 values are given in online supplementary table S1. Agreement between the duplicate KLK8 measurements was assessed graphically using Bland-Altman plots and scatter plots (see online supplementary figure 1). Associations between KLK8 and $A \beta_{42}$ and P-/T-tau were assessed using Spearman's rank correlation coefficient. The diagnostic performance of KLK8 was determined using receiver operating characteristic (ROC) analyses. Optimal cut-off values for KLK8 were based on the Youden index. Specificity, sensitivity, the ORs and accuracy corresponding to the optimal cut-off points were calculated; the latter was calculated as the fraction of all patients who were correctly categorised (ie, (true positives + true negatives)/number of participants). The area under the ROC curve (AUC) and its associated 95\% CI were used to assess discriminative performance of KLK8 in general. Adjusted ROC analyses were based on the predictive probabilities of a generalised linear mixed model (GLMM) with Bernoulli distribution function, logit link function and variance component covariance structure. The GLMM included the biomarker of interest (ie, KLK8, $\mathrm{A} \beta_{42}$ P-tau, T-tau and/or $\mathrm{A} \beta_{42} / \mathrm{A} \beta_{40}$ ratio) as well as age and gender as fixed effects and study group/site as random effect. In a sensitivity analysis, we excluded the study group B or included only subgroups of controls, that is (a) only healthy volunteers and headache patients or (b) only patients with psychiatric diseases and PD into the ROC analyses. As the number of the patients in these subgroups was considerably smaller, we could only estimate unadjusted AUC and 95\% CIs because adjusted models did not converge. Furthermore, added value of CSF or blood KLK8 on diagnostic accuracy of CSF AD core biomarkers were evaluated by assessing the adjusted AUC values and 95\% CIs. Statistical differences between AUCs were assessed using the DeLong test. All tests were two-tailed at a significance level of $\alpha=0.05$. All data were analysed using the software package SPSS V.22 (IBM) and SAS statistical analysis system V.9.4 

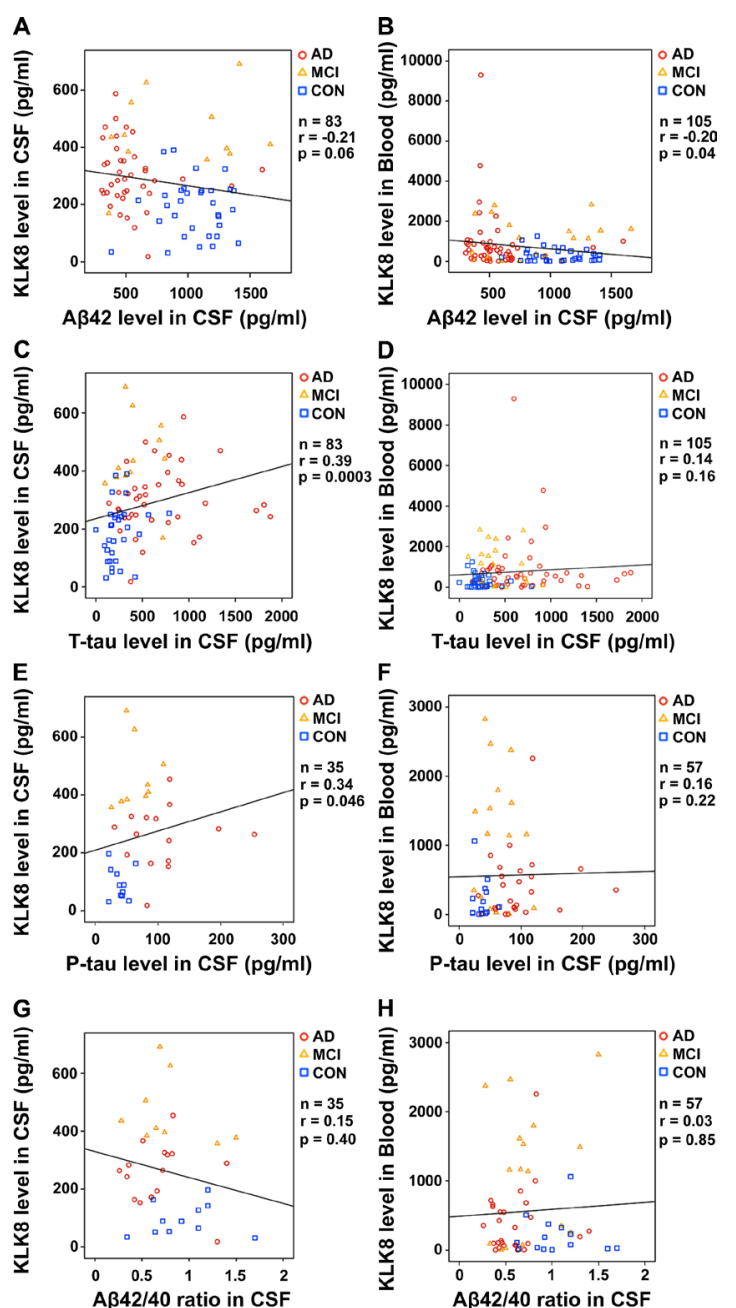

P-tau level in CSF $(\mathrm{pg} / \mathrm{ml})$
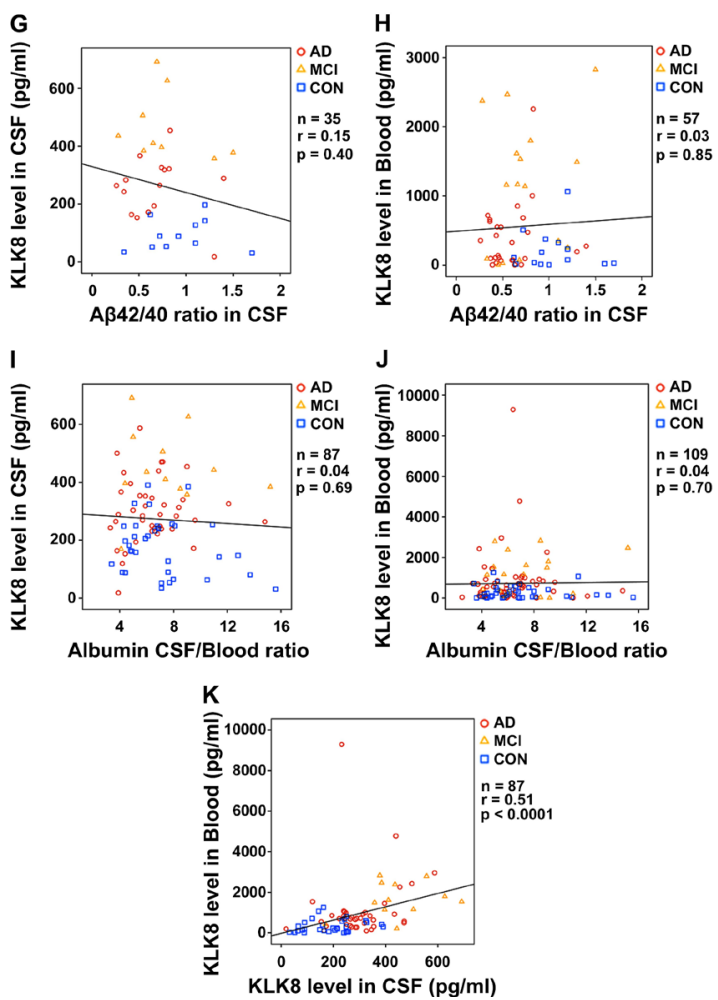

Figure 3 Associations between KLK8 in CSF and blood and CSF AD biomarkers correlation analyses were assessed using Spearman's rank correlation coefficients and their corresponding $p$ values. AD, Alzheimer's disease; $A \beta_{42 \text { or } 40} \beta$-amyloid 42 or 40 ; CON, controls; CSF, cerebrospinal fluid; KLK8, Kallikrein-8; $M C l$, mild cognitive impairment due to $A D ; P$-tau, phosphorylated tau; $P$, p value; r, Spearman's rank correlation coefficient; T-tau, total tau.

For detailed information about blood and CSF sampling, APOE genotyping, A $\beta 42$, T-/P-tau as well as KLK8 measurements, see online supplementary methods.
Data availability statement

Any anonymised data not published in the article will be shared on request from any qualified investigator.

\section{RESULTS}

\section{Data set description}

Demographic, genetic and biochemical data of all groups are shown in table 1. AD and MCI patients were older (72 years (64-77) than controls (64 years (55-68)). The female:male ratios for AD, MCI and controls were $1.5: 1,1: 2.3$ and $1: 1$, respectively.

\section{CSF and blood KLK8 levels in AD and $\mathrm{MCl}$ patients versus controls}

In comparison to controls, $\mathrm{AD}$ and $\mathrm{MCI}$ samples showed significantly higher levels of KLK8 in CSF (AD: 286.3 pg/mL (239.8353.4) versus control: $181.8 \mathrm{pg} / \mathrm{mL}(88.7-248.5), \mathrm{p}<0.00001$; MCI: $423.0 \mathrm{pg} / \mathrm{mL}$ (379.3-543.9) versus control: $181.8 \mathrm{pg} /$ $\mathrm{mL}(88.7-248.5), \mathrm{p}<0.00001)$ (figure $2 \mathrm{~A}$ ) and in blood (AD: $453.9 \mathrm{pg} / \mathrm{mL}(146.5-719.6)$ versus control: $227 \mathrm{pg} / \mathrm{mL}$ (48.3508.4), $\mathrm{p}=0.00003$; MCI: $1139.3 \mathrm{pg} / \mathrm{mL}(90.6-1705.8)$ versus control: $227 \mathrm{pg} / \mathrm{mL}$ (48.3-508.4), $\mathrm{p}=0.003$ ) (figure 2B). Of note, MCI patients had the highest KLK8 levels, also in comparison to AD patients. Even after the exclusion of blood KLK8 outlier values in the $\mathrm{AD}$ group the difference to control remained highly significant $(p<0.0003)$. Within the control population, CSF and/ or blood KLK8 levels of patients with PD or with psychiatric diseases were significantly lower than the values of healthy volunteers or patients with headache (figure 2C,D), while the KLK8 values among the latter two groups were in a similar range (figure 2D). But even after exclusion of the patients with psychiatric diagnoses and PD from the control population the CSF or blood KLK8 values of $\mathrm{AD}$ or MCI patients remained higher than control participants (ie, healthy volunteers and patients with tension-type headache but otherwise healthy) $(\mathrm{p} \leq 0.037)$ (data not shown).

There was a strong variation between blood KLK8 levels of study group B and that of other study groups (study group B: 91.6 pg/mL (30.0-388.6), study groups A, C and D: $545.3 \mathrm{pg} / \mathrm{mL}$ (238.8-991.2), $\mathrm{p}=0.00001)$. This was presumably due to differences in preanalytic procedures and in particular due to the blood storage length (study group B: 7 (4-10.5) and in study groups A, $\mathrm{C}$ and D: 46 (44-60) months). There were no noteworthy differences in CSF and blood KLK8 levels among study groups/sites A, C and $\mathrm{D}$ when comparing the same diagnostic groups. Neither CSF nor blood KLK8 levels revealed any significant gender specific differences within the diagnostic groups (data not shown).

\section{KLK8 correlation with other biochemical, clinical and genetic} parameters

To evaluate the associations between KLK8 and different laboratory data correlation analyses were performed (figure 3). CSF and blood KLK8 showed both a weak negative correlation with CSF $A \beta_{42}$ (figure $3 \mathrm{~A}, \mathrm{~B}$ ). There was a weak positive correlation between CSF KLK8 and CSF T-tau (figure 3C) as well as P-tau (figure 3E) but barely any between blood KLK8 and CSF T-tau (figure 3D) or CSF P-tau (figure 3F). No correlations were found between CSF or blood KLK8 values and CSF $\mathrm{A} \beta_{42} / \mathrm{A} \beta_{40}$ ratio (figure $3 \mathrm{G}, \mathrm{H}$ ) or albumin $\mathrm{CSF} /$ blood ratio (figure $3 \mathrm{I}, \mathrm{J}$ ). The strongest correlation was detected between CSF and blood KLK8 (figure $3 \mathrm{~K}$ ), while no correlations were detectable with APOE genotype (CSF: $n=26, r=-0.11, p=0.60$, blood: $n=58$, $\mathrm{r}=0.13, \mathrm{p}=0.32$ ) or gender (CSF: $\mathrm{n}=87, \mathrm{r}=-0.07, \mathrm{p}=0.5$, blood: $\mathrm{n}=237, \mathrm{r}=-0.004, \mathrm{p}=0.95)$. There was a weak to 
Table 2 Diagnostic accuracy of CSF and blood KLK8 (unadjusted analyses)

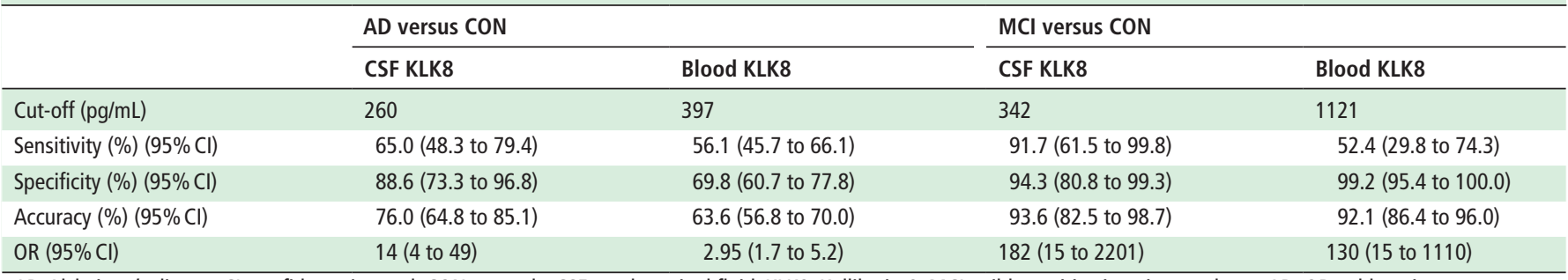

$A D$, Alzheimer's disease; $C l$, confidence interval; $C O N$, controls; CSF, cerebrospinal fluid; KLK8, Kallikrein-8; $M C l$, mild cognitive impairment due to $A D ; O R$, odds ratio.

moderate positive correlation between age and KLK8 levels in blood $(n=237, r=0.13, p=0.04)$ and $\operatorname{CSF}(n=87, r=0.42$, $\mathrm{p}=0.00005)$, respectively. However, the association between age and KLK8 was at least in part explicable by different age distributions among control subjects on one hand and AD and MCI patients on the other hand, therefore presumably a biassed one. When looking at CON group or $\mathrm{AD}+\mathrm{MCI}$ group separately, no age-related correlations were seen (data not shown).

\section{Diagnostic accuracies of CSF and blood KLK8}

In unadjusted ROC analyses CSF KLK8 showed moderately high or high AUCs for AD versus controls 0.80 (95\% CI 0.70 to 0.90 ) and for MCI versus controls 0.94 (95\% CI 0.86 to 1.0$)$. The optimal cut-offs (according to the Youden index) for CSF KLK8 to distinguish $\mathrm{AD}$ and MCI patients from individuals without cognitive deficits were $\geq 260$ and $\geq 342 \mathrm{pg} / \mathrm{mL}$, respectively. Presumably due to large inter-cohort differences of KLK8 blood serum values, blood KLK8 showed lower AUCs for AD versus control 0.67 (95\% CI 0.60 to 0.74 ) and for MCI versus control 0.70 (95\% CI 0.55 to 0.85 ) in comparison to CSF KLK8. The optimal cut-offs for blood KLK8 were $\geq 397 \mathrm{pg} / \mathrm{mL}$ (for AD) and $\geq 1121 \mathrm{pg} / \mathrm{mL}$ (for MCI). Sensitivity, specificity, accuracy and ORs are shown in table 2.

There was a considerable heterogeneity among the control participants being compiled of healthy volunteers $(n=50)$, patients with tension-type headache $(n=21)$, with PD $(n=19)$, or with psychiatric diagnoses $(n=28)$. In order to evaluate the influence of the composition of controls on the ability of KLK8 to correctly allocate participants to different diagnostic groups sensitivity analyses for the unadjusted AUCs were performed (table 3). When control group was restricted to patients with psychiatric disorders and PD, the discrimination slightly improved for both, CSF and blood KLK8 (by relative change of $5.3 \%$ up to $20.0 \%$, depending whether it was measured in CSF or blood and was applied to discriminate MCI or AD from controls), while restricting control group to healthy volunteers and patients with tension-type headache slightly worsened the discrimination (by $3.3 \%$ up to $12.5 \%$ ). Blood samples of the study group B had the shortest storage time (see figure 1) and the lowest KLK8 values when compared with all other study groups (median in B: $91.6 \mathrm{pg} / \mathrm{mL}$, median in A, C, D: $545.3 \mathrm{pg}$ / $\mathrm{mL}$ ). Excluding the study group $\mathrm{B}$ from the analysis improved the ability of blood KLK8 to discriminate AD and MCI patients from controls by $4.5 \%$ up to $25.7 \%$. It should be emphasised that AUC values of the sensitivity analyses are not adjusted and adjustment in the main analysis was associated with an increase of the AUC.

After adjusting for age, gender and study site the discrimination performance of blood KLK8 for AD vs control $0.83(95 \%$ CI 0.74 to 0.91 ) (figure $4 \mathrm{~A}$ ) and MCI vs control $0.94(95 \% \mathrm{CI}$ 0.86 to 1.0 ) (figure $4 \mathrm{~B}$ ) improved by $24 \%$ and $34 \%$, respectively; while the already high performance of CSF KLK8 became only slightly better by $11 \%$ for AD versus control 0.89 (95\% CI 0.81 to 0.97 ) (figure 4C) and by $3 \%$ for MCI versus control 0.97 (95\% CI 0.91 to 1.0 ) (figure 4D). The performance of CSF KLK8 in distinguishing $\mathrm{AD}$ from control was similar to that of CSF $\mathrm{A} \beta_{42}(\mathrm{p}=0.36)$ and T-tau $(\mathrm{p}=0.19)$ (figure $\left.4 \mathrm{C}\right)$. The performance of CSF KLK8 for discrimination of MCI and control was even superior to that of CSF A $\beta_{42}(p=0.04)$ but not T-tau $(p=0.12)$ (figure 4D). The adjusted AUC of blood KLK8 for MCI versus controls was also comparable to those of CSF $A \beta_{42}(p=0.13)$ and T-tau $(\mathrm{p}=0.34)$ (figure $4 \mathrm{~B})$, while the AUC of blood KLK8 for $\mathrm{AD}$ versus control was slightly lower than those of CSF $A \beta_{42}$ $(p=0.006)$ and T-tau $(p=0.007)$ (figure $4 A)$.

When restricting the analysis to samples with available data for CSF P-tau and CSF $A \beta_{42} / A \beta_{40}$ ratio, KLK8 AUCs changed slightly (figure $4 \mathrm{E}-\mathrm{H}$ ). When discriminating $\mathrm{AD}$ versus control the AUC of blood KLK8 $(0.74 ; 95 \%$ CI 0.57 to 0.91$)$ was lower than that of CSF P-tau $(p=0.005)$ and $A \beta_{42} / A \beta_{40}$ ratio $(p=0.03)$ (figure $4 \mathrm{E}$ ), while the AUC of blood KLK8 for MCI versus

Table 3 Sensitivity analyses for unadjusted AUCS

\begin{tabular}{|c|c|c|c|c|c|}
\hline & & \multicolumn{4}{|c|}{ AUC $(95 \% \mathrm{CI})$ (unadjusted analyses) } \\
\hline & & $\begin{array}{l}\text { All AD patients } \\
\text { All } \mathrm{MCl} \text { patients } \\
\text { All CON participants }\end{array}$ & $\begin{array}{l}\text { All AD patients } \\
\text { All } \mathrm{MCl} \text { patients } \\
\text { CON: healthy and headache only }\end{array}$ & $\begin{array}{l}\text { All AD patients } \\
\text { All } \mathrm{MCl} \text { patients } \\
\text { CON: psychiatric and PD only }\end{array}$ & $\begin{array}{l}\text { All participants minus } \\
\text { study group B }\end{array}$ \\
\hline \multirow[t]{2}{*}{$A D$ versus $C O N$} & CSF KLK8 & $0.80(0.70$ to 0.90$)$ & $0.70(0.55$ to 0.83$)$ & $0.96(0.90$ to 1.0$)$ & $0.80(0.70$ to 0.90$)$ \\
\hline & Blood KLK8 & $0.67(0.60$ to 0.74$)$ & $0.60(0.51$ to 0.68$)$ & $0.76(0.67$ to 0.85$)$ & $0.70(0.62$ to 0.77$)$ \\
\hline \multirow[t]{2}{*}{$\mathrm{MCl}$ versus $\mathrm{CON}$} & CSF KLK8 & $0.94(0.86$ to 1.0$)$ & $0.91(0.77$ to 1.0$)$ & $0.99(0.98$ to 1.0$)$ & $0.94(0.86$ to 1.0$)$ \\
\hline & Blood KLK8 & $0.70(0.55$ to 0.85$)$ & 0.65 (0.48 to 0.83$)$ & 0.77 (0.64 to 0.90$)$ & $0.88(0.74$ to 1.0$)$ \\
\hline
\end{tabular}

AUCS of CSF and blood KLK8 for discrimination of AD or MCl from controls are shown for different subgroups of participants. The first column shows data for all participants. In the second column patients with psychiatric diseases or PD and in the third column patients with tension-type headache and healthy volunteers are excluded. The fourth column represents the AUC values for all participants after exclusion of study group B (with blood samples only). Please note that AUC values of the sensitivity analyses are not adjusted and that adjustment in the main analysis was associated with an increase of the AUC.

$A D$, Alzheimer's disease; AUC, area under the curve; $\mathrm{Cl}$, confidential interval; $\mathrm{CON}$, controls; CSF, cerebrospinal fluid; Headache, patients with tension-type headache; Healthy, healthy volunteers; KLK8, Kallikrein-8; MCI, mild cognitive impairment due to AD;PD, Parkinson's disease; Psychiatric, patients with psychiatric diagnoses. 
A AD vs. CON (by Blood KLK8)
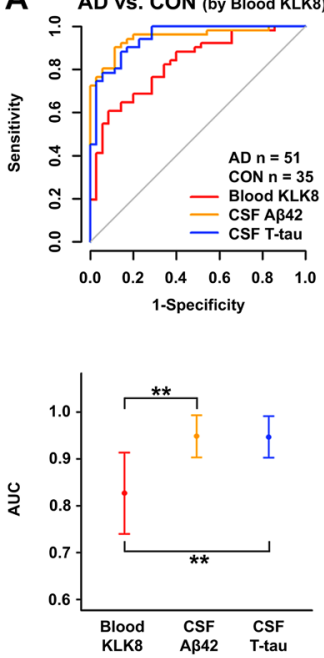

E AD vs. CON (by Blood KLK8)
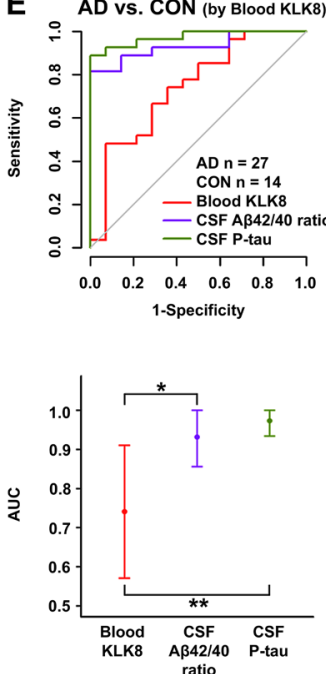

B $\mathrm{MCl}$ vs. CON (by Blood KLK8)
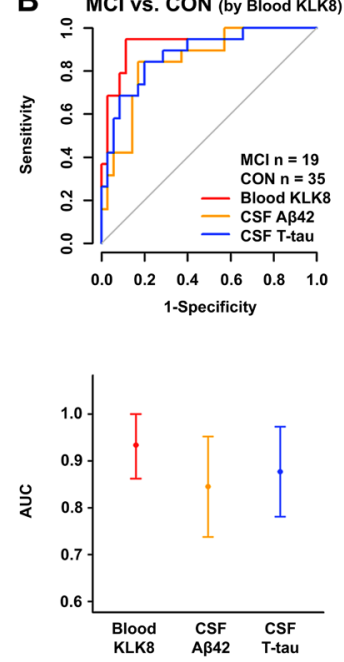

F MCl vs. CON (by Blood KLK8)
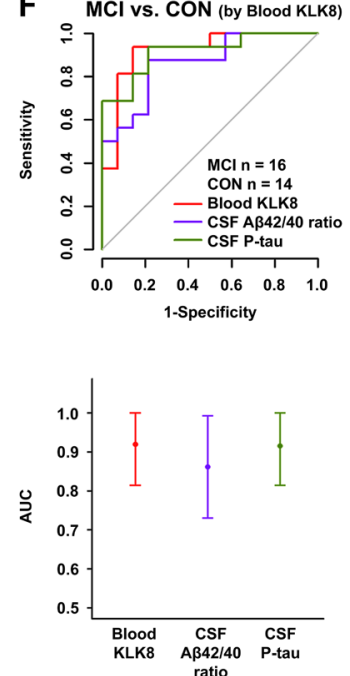

C AD vs. CON (by CSF KLK8)
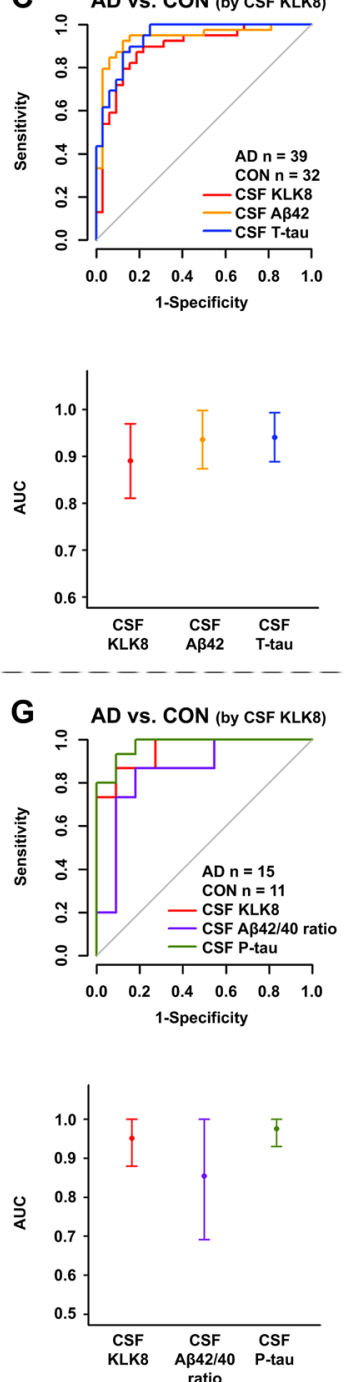

D $\mathrm{MCl}$ vs. CON (by CSF KLK8)
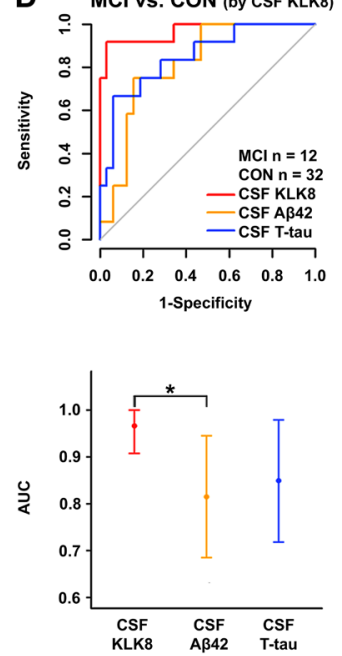

H MCl vs. CON (by CSF KLKE)
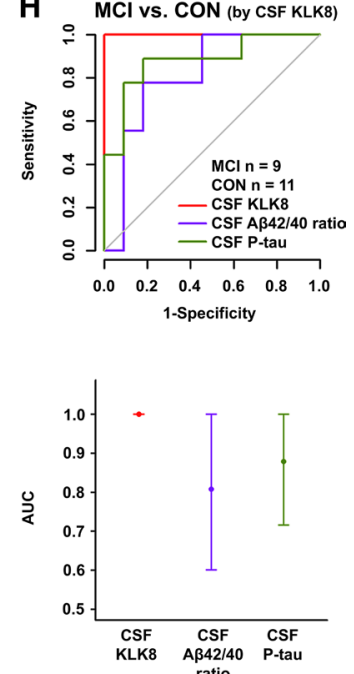

Figure 4 Diagnostic performance of KLK8 in comparison to CSF AD biomarkers ROC analyses (upper panel) and AUC plots (lower panel) of CSF T-tau and CSF $A \beta_{42}(A-D)$ or CSF P-tau and CSF A $\beta_{42} / A \beta_{40}$ ratio $(E-H)$ in comparison to KLK8 in blood $(A, B, E, F)$ and $C S F(C, D, G, H)$. A and $C$ show the performances of blood (A) and CSF (C) KLK8 as well as that of CSF T-tau and CSF A $\beta_{42}$ in distinguishing AD from controls; the performances for discrimination of $\mathrm{MCl}$ and control are represented in B and D. The performances of blood (E) and CSF (G) KLK8 for discriminating AD from controls in comparison to CSF P-tau and CSF $A \beta_{42} / A \beta_{40}$ are shown in $E$ and $G$, while the performances for distinguishing $\mathrm{MCl}$ from controls are represented in $\mathrm{F}$ and $\mathrm{H}$. Data were adjusted for gender, age and study group. Point estimates in the lower panel represent AUCs and the error bars each $95 \% \mathrm{CI}$. P values were determined by DeLong test. For the sake of better comparability with other biomarkers ROC curves for CSF $A \beta_{42}$ and CSF A $\beta_{42 / 40}$ are depicted as '-CSF A $\beta_{42}{ }^{\prime}$ and '-CSF A $\beta_{42 / 40}{ }^{\prime}$. ${ }^{*} P \leq 0.04$; ${ }^{* *} p \leq 0.007$. AD, Alzheimer's disease; AUC, area under the curve; $A \beta_{42 \text { or } 40^{\prime}} \beta$-amyloid 42 or 40 ; CON, controls; CSF, cerebrospinal fluid; $M C l$, mild cognitive impairment due to $A D$; P-tau, phosphorylated tau; ROC, receiver operating characteristic; T-tau, total tau.

control (AUC: $0.92 ; 95 \%$ CI 0.81 to 1.0 ) was similar to that of CSF $A \beta_{42} / A \beta_{40}$ ratio $(p=0.40)$ and P-tau $(p=0.94)$ (figure $\left.4 F\right)$. The performance of CSF KLK8 for AD versus control (AUC: $0.95 ; 95 \%$ CI 0.88 to 1.0 ) was comparable to that of CSF P-tau $(\mathrm{p}=0.56)$ and $\mathrm{A} \beta_{42} / \mathrm{A} \beta_{40}$ ratio $(\mathrm{p}=0.20)$ (figure $\left.4 \mathrm{G}\right)$ and for $\mathrm{MCI}$ versus control (AUC: $1.0 ; 95 \%$ CI 1.0 to 1.0 ) even better than that of CSF $A \beta_{42} / A \beta_{40}$ ratio $(p=0.07)$ but similar to that of CSF P-tau $(\mathrm{p}=0.15)$ (figure $4 \mathrm{H})$.

In a next step, the added values of KLK8 in CSF and blood on diagnostic accuracy of CSF AD biomarkers were evaluated (table 4). Given that the diagnostic performances of CSF core biomarker were already excellent for the discrimination of $\mathrm{AD}$ from control (all AUCs >0.90), there was no additive effect of KLK8 measurement. When differentiating MCI from control, the diagnostic accuracy of the combination of CSF KLK8 and
CSF $A \beta_{42}$ was significantly better than that of CSF $A \beta_{42}$ alone $(\mathrm{p}=0.03)$.

\section{DISCUSSION}

This explorative study is the first to evaluate the diagnostic performance of CSF and blood KLK8 for AD. KLK8 is a relatively unknown player within the setting of $\mathrm{AD}$. This extracellular serine protease was first related to $\mathrm{AD}$ by showing its mRNA upregulation in disease-affected human hippocampus. ${ }^{20}$ Interestingly, single nucleotide polymorphisms in CD33, TOMM40, ${ }^{21} \mathrm{APOE}^{22}$ and as recently shown also $\mathrm{KLK}^{23}$ are located in the same chromosomal region $19 \mathrm{q} 13$ which seems to be strongly associated with AD risk. While different KLK8 substrates, that is, ephrin receptor $\mathrm{B} 2,{ }^{24}$ fibronectin, ${ }^{25}$ neuregulin- $1^{26}$ and L1-CAM ${ }^{27}$ have been shown to be directly involved 
Table 4 Added value of CSF and blood KLK8 on diagnostic accuracy of CSF AD biomarkers

\begin{tabular}{|c|c|c|c|c|c|c|c|c|}
\hline & \multicolumn{4}{|l|}{$A D$ versus $C O N$} & \multicolumn{4}{|l|}{$\mathrm{MCl}$ versus $\mathrm{CON}$} \\
\hline & \multicolumn{2}{|l|}{ CSF KLK8 } & \multicolumn{2}{|l|}{ Blood KLK8 } & \multicolumn{2}{|l|}{ CSF KLK8 } & \multicolumn{2}{|l|}{ Blood KLK8 } \\
\hline & AUC $(95 \% \mathrm{Cl})$ & $P$ value & AUC $(95 \% \mathrm{Cl})$ & $P$ value & AUC $(95 \% \mathrm{Cl})$ & $P$ value & AUC $(95 \% \mathrm{Cl})$ & $P$ value \\
\hline$A \beta_{42}$ & $0.94(0.87$ to 1.0$)$ & & $0.96(0.92$ to 1.0$)$ & & 0.88 (0.78 to 0.98 ) & & 0.90 (0.82 to 0.98 ) & \\
\hline$A \beta_{42}+K L K 8$ & $0.97(0.94$ to 1.0$)$ & & $0.97(0.92$ to 1.0$)$ & & 0.99 (0.96 to 1.0$)$ & & $0.96(0.92$ to 1.0$)$ & \\
\hline AUC difference & $0.04(-0.02$ to 0.09$)$ & 0.20 & $0.01(-0.03$ to 0.05$)$ & 0.59 & 0.11 (0.01 to 0.21$)$ & 0.03 & $0.06(-0.01$ to 0.13$)$ & 0.06 \\
\hline T-tau & 0.94 (0.89 to 0.99$)$ & & 0.95 (0.91 to 0.99$)$ & & 0.94 (0.87 to 1.0$)$ & & 0.95 (0.90 to 1.0$)$ & \\
\hline T-tau +KLK8 & $0.96(0.91$ to 1.0$)$ & & $0.97(0.93$ to 1.0$)$ & & $1.0(1.0$ to 1.0$)$ & & $1.0(1.0$ to 1.0$)$ & \\
\hline AUC difference & $0.02(-0.02$ to 0.05$)$ & 0.32 & $0.01(-0.01$ to 0.04$)$ & 0.36 & $0.06(-0.01$ to 0.13$)$ & 0.11 & $0.05(-0.01$ to 0.1$)$ & 0.07 \\
\hline P-tau & $0.98(0.93$ to 1.0$)$ & & $0.97(0.93$ to 1.0$)$ & & $0.88(0.72$ to 1.0$)$ & & 0.91 (0.81 to 1.0 ) & \\
\hline P-tau +KLK8 & $1.0(1.0$ to 1.0$)$ & & 0.97 (0.93 to 1.0$)$ & & $1.0(1.0$ to 1.0$)$ & & $0.98(0.95$ to 1.0$)$ & \\
\hline AUC difference & $0.02(-0.02$ to 0.07$)$ & 0.30 & $-0.003(-0.02$ to 0.01$)$ & 0.69 & $0.12(-0.04$ to 0.28$)$ & 0.15 & $0.07(-0.03$ to 0.18$)$ & 0.18 \\
\hline $\mathrm{A} \beta_{42}+\mathrm{T}$ tau $+\mathrm{P}$-tau & $0.97(0.91$ to 1.0$)$ & & $0.98(0.96$ to 1.0$)$ & & $0.91(0.76$ to 1.0$)$ & & $0.92(0.81$ to 1.0$)$ & \\
\hline $\mathrm{A} \beta_{42}+\mathrm{T}$ tau+P tau+KLK8 & $1.0(1.0$ to 1.0$)$ & & $1.0(1.0$ to 1.0$)$ & & $1.0(1.0$ to 1.0$)$ & & $1.0(1.0$ to 1.0$)$ & \\
\hline AUC difference & $0.03(-0.03$ to 0.09$)$ & 0.29 & $0.02(-0.01$ to 0.04$)$ & 0.25 & $0.09(-0.06$ to 0.24$)$ & 0.23 & $0.08(-0.02$ to 0.19$)$ & 0.10 \\
\hline
\end{tabular}

AUCs of CSF AD core biomarkers (ie, CSF A $\beta_{42^{\prime}}$ CSF T-tau, CSF P-tau each and combination of all three) are compared with AUCS of CSF AD biomarker of interest with added value of CSF or blood KLK8. Data were adjusted for gender, age and study group as well as for biomarker of interest. p values were determined by DeLong test.

$A \beta_{42^{\prime}} \beta$-amyloid 42; $A D$, Alzheimer's disease; $A U C$, area under the curve; $C O N$, controls; $C S F$, cerebrospinal fluid; $M C l$, mild cognitive impairment due to $A D ; P, p$ value; $P$-tau, phosphorylated tau; T-tau, total tau.

in the pathomechanism of AD, KLK8 itself has been neglected until recently. Our lab was the first to examine the role of KLK8 in the context of $\mathrm{AD}$ in detail. ${ }^{17}$ We could demonstrate excessive KLK8 mRNA and protein levels at incipient AD stages in murine and human brain. Furthermore, we could show that short-term inhibition of cerebral KLK8 by an anti-KLK8 antibody at a moderate stage disease was capable of mitigating multiple features of Alzheimer's pathology in transgenic mice. KLK8 blockade reduced for instance $A \beta$ pathology by impeding amyloidogenic amyloid precursor protein processing, facilitating $A \beta$ clearance across the blood-brain-barrier and boosting microglial $A \beta$ phagocytosis and autophagy, resulting in decreased $\mathrm{A} \beta$ plaque burden, improved spatial memory performance, and reduced anxiety. ${ }^{17}$

In line with our previous results, we now show also in CSF and blood of $\mathrm{AD}$ and MCI patients higher KLK8 levels, with MCI patients showing the highest values. The diagnostic accuracy of CSF KLK8 was at least as good as CSF A $\beta_{42}$, T-tau and P-tau for both MCI and AD and in case of MCI even better than CSF A $\beta_{42}$. Blood KLK8 was a similarly good discriminator for MCI but slightly worse for $\mathrm{AD}$ when compared with the aforementioned core CSF biomarkers. When comparing the diagnostic accuracy of KLK8 with CSF A $\beta_{42}$, P-tau and T-tau one should bear in mind that those established AD biomarkers were (beside neuropsychological test results) essential components of the diagnostic criteria; yet, their partial superiority over KLK8 is a biassed one.

Interestingly, KLK8 levels were higher in MCI (in CSF approx. 1.5 -fold and in blood 2.5 -fold) than in AD patients, its diagnostic accuracy was better for MCI (AUC in CSF $=0.97$ and blood $=0.94$ ) than for $\mathrm{AD}$ (AUC in $\mathrm{CSF}=0.89$, blood $=0.83$ ), and there was an added value of CSF KLK8 (to the core CSF biomarkers) for MCI (table 4). The better performance of KLK8 in $\mathrm{MCI}$ compared with $\mathrm{AD}$ group might be an advantage over the established core biomarkers with their relatively stable levels during the disease course, possibly qualifying KLK8 as a better diagnostic tool in early stage disease.

The composition of the control participants was heterogeneous, consisting of healthy volunteers, patients with tensiontype headache, PD and psychiatric disorders. This heterogeneity is not necessarily a weakness since it mirrors a more realistic population in clinical practice. Of note, the diagnostic accuracy of KLK8 became in the sensitivity analysis slightly worse (by $3.2 \%$ up to $12.5 \%$, depending whether it was measured in CSF or blood and was used to discriminate MCI or AD from control, see table 3) when the control population was restricted to healthy volunteers and patients with headache (ie, after exclusion of patients with PD or psychiatric diagnoses). This finding is very well explicable by the fact that control patients with PD and psychiatric diseases had the lowest CSF and blood KLK8 levels in comparison to all other diagnostic groups. The median blood KLK8 value of these patients was four times lower than that of healthy volunteers or participants with headache and six or 15 times lower in comparison to $\mathrm{AD}$ or $\mathrm{MCI}$, respectively. Obviously, KLK8 increase does not reflect an unspecific change occurring during CNS damage/neurodegeneration; but whether it is specific to $\mathrm{AD}$ and-in contrast to the established core biomarkers ${ }^{67}$-capable of distinguishing $\mathrm{AD}$ from other neurodegenerative dementias remains to be clarified.

As recently shown ${ }^{28}$ KLK8 levels seem to be higher in AD affected and non-affected female murine and human brain when compared with male individuals, while contrastingly no correlations were found between CSF or blood KLK8 levels and gender in this work. No significant correlations were also detectable between CSF or blood KLK8 values and APOE genotype. Given that APOE status was available only for a small subgroup of participants $(\mathrm{n}=58)$, these results, however, must be viewed cautiously.

The source of blood KLK8 is not known. KLK8 is produced by various organs, ${ }^{29}$ thus it is unclear whether higher levels of blood KLK8 affect or are affected by CNS KLK8. In either way, this study provides strong evidence that CSF and blood KLK8 concentrations both might be associated with AD.

There is now general agreement that $\mathrm{AD}$ has polygenic origins with multiple drivers that trigger the progression of neurodegeneration. Hence, there is an urgent need to identify additional biomarkers that might also help us to better understand the complex heterogeneity of the disease and uncover novel pathogenic pathways. Without exception, all known biomarkers relay the old and well known pathophysiological AD story-be 
it the established core biomarkers that represent the hallmarks of the disease, as currently defined, or the putative biomarkers, such as synapse dysfunction biomarkers (Neurogranin, SNAP25, Synaptotagmin), neuronal cell death biomarkers (VILIP-1, NF-L), inflammation/microglial activation biomarkers (IL-6, TNF-alpha, TGF-beta, TREM2), vascular dysfunction biomarker (hFABP), iron toxicity biomarker (Ferritin) and so on (for an overview $\mathrm{se}^{30}$ ) that reflect ancillary downstream changes triggered by $A \beta$ and tau pathology. KLK8 is different. This extracellular serine protease appears to have-based on what we hitherto know-no direct interaction with amyloid or tau metabolism. Its rise in $\mathrm{AD}$ does not represent a downstream event; it is up-regulated in $\mathrm{AD}$-affected human brain already when only the first speck of amyloid deposition is histologically visible and in transgenic mouse brain the upregulation is detectable even prior to any amyloid deposition. Moreover, KLK8 is not only a potential indicator of $\mathrm{AD}$; its inhibition exerts also versatile therapeutic effects in transgenic mouse. Together, these findings now offer a glimmer of hope that KLK8 measurements in CSF and/or blood could someday become a diagnostic tool for patients' stratification and personalised treatment.

The present study has some limitations. The population size with 237 participants in total was relatively small, and CSF samples from many control individuals and some AD or MCI patients were lacking (see figure 1), thus the diagnostic accuracy of CSF and blood KLK8 in comparison to performances of core CSF biomarkers were restricted to a relatively small participants' subgroup. The composition of our control group may have been a source for Berksonian bias. Indeed, our sensitivity analyses have shown that excluding the patients with PD or psychiatric diagnoses from the controls worsen and restricting the controls to these patients improves the AUC values (see table 3), but in any case, blood and CSF KLK8 values remain a good to excellent discriminator for $\mathrm{AD}$ or $\mathrm{MCI}$ versus control. Given that in the daily clinical life the target population includes both neurologically/psychiatrically healthy and diseased patients, we believe that the analyses including all of our control subgroups have produced the most valid values. Owing to the fact that our data set did not include longitudinal follow-up, it was not possible to distinguish between stable MCI and those progressing to dementia. However, all of our MCI patients had according to NIA-AA criteria ${ }^{18}$ a high likelihood to convert to AD. All clinical diagnoses were first time diagnoses and apart from them none of the participants revealed any noteworthy diseases. Nonetheless a confounding effect of an unknown comorbidity or premedication cannot be entirely excluded. Collection of blood and CSF samples were not performed in one central reference laboratory and the storage duration of the samples differed among the study groups in part considerably, explaining at least in part the variability of KLK8 values, especially in blood, across study sites. Although our analyses were adjusted for centre effects, the relatively large differences between the study groups $\mathrm{B}$ on one hand and $\mathrm{A}, \mathrm{C}$ and D (figure 1) on the other could have still been a source of distortion. Lack of standardised preanalytical methods across study centres and laboratories is a well-known problem when searching for biomarkers ${ }^{31-33}$-an issue which has to be overcome for future studies. Optimum cut-off values can only be established under standardised preanalytic and postanalytic procedures. The KLK8 cut-off values (table 2) in this work are obviously not suggested for clinical use but were instead provided to evaluate the potential KLK8 functionality as a biomarker.

To conclude, these findings suggest that both CSF and blood KLK8 may serve as a novel biomarker for diagnosis of $\mathrm{AD}$ at incipient stages. Future prospective validation studies in larger cohorts are warranted.

\section{Author affiliations}

${ }^{1}$ Institute of Neuropathology, Faculty of Medicine, University of Duisburg-Essen, Essen, Germany

${ }^{2}$ Institute of Medical Informatics, Biometry and Epidemiology, Faculty of Medicine, University of Duisburg-Essen, Essen, Germany

${ }^{3}$ Department of Psychiatry and Psychotherapy, University Medical Center, Gottingen, Germany

${ }^{4}$ Department of Neurology, University of Ulm, Ulm, Germany

${ }^{5}$ Clinic for Neurogeriatrics and Neurological Rehabilitation, RKU-University and Rehabilitation Hospital Ulm, Ulm, Germany

${ }^{6}$ Department of Neurology, RWTH Aachen University, Aachen, Germany

${ }^{7}$ Center of Clinical Epidemiology, c/o Institute of Medical Informatics, Biometry and Epidemiology, Faculty of Medicine, University of Duisburg-Essen, Essen, Germany

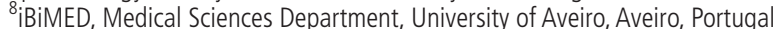

Acknowledgements We thank Michaela Knoll (Essen) and Julia Heller (Aachen) for excellent technical assistance.

Contributors Study concept and design: AH, KK. Acquisition, analysis or interpretation of data: all authors. Drafting of manuscript: ST-H, KK. Critical revision of the manuscript for important intellectual content: all authors. Statistical analysis: JR, K-HJ, ST-H, KK. Funding: AH, KK. Administrative, technical or material support: ST-H, AH, JR, JV, JW, KR, MO, CVA, HT, KK. Study supervision: KK.

Funding This study was supported by a grant (to Arne Herring and Kathy Keyvani) from European Regional Development Fund (ERDF-0400098).

Competing interests $\mathrm{AH}$ and $\mathrm{KK}$ are inventors on the pending patent 'Agents inhibiting Kallikrein-8 for use in the prevention or treatment of Alzheimer's disease', which is registered at the European Patent Agency since 09/2015 (EP 15003657/15003657.2) and at the US Patent and Trademark Office since 03/2018 $(15 / 761,725)$.

\section{Patient consent for publication Not required.}

Ethics approval Measurement of KLK8 in the anonymised samples was approved by the ethics committee of the Medical Faculty, University of Duisburg-Essen, Germany (16-7182-BO). This study followed the tenets of the current version of the Declaration of Helsinki.

Provenance and peer review Not commissioned; externally peer reviewed.

Data availability statement All data relevant to the study are included in the article or uploaded as supplementary information.

Open access This is an open access article distributed in accordance with the Creative Commons Attribution Non Commercial (CC BY-NC 4.0) license, which permits others to distribute, remix, adapt, build upon this work non-commercially, and license their derivative works on different terms, provided the original work is properly cited, appropriate credit is given, any changes made indicated, and the use is non-commercial. See: http://creativecommons.org/licenses/by-nc/4.0/.

\section{ORCID iDs}

Kathrin Reetz http://orcid.org/0000-0002-9730-9228

Markus Otto http://orcid.org/0000-0002-6647-5944

Kathy Keyvani http://orcid.org/0000-0003-2558-5159

\section{REFERENCES}

1 Bateman RJ, Xiong C, Benzinger TLS, et al. Clinical and biomarker changes in dominantly inherited Alzheimer's disease. N Engl J Med 2012;367:795-804.

2 Jack CR, Knopman DS, Jagust WJ, et al. Tracking pathophysiological processes in Alzheimer's disease: an updated hypothetical model of dynamic biomarkers. Lancet Neurol 2013;12:207-16.

3 Palmqvist $\mathrm{S}$, Zetterberg $\mathrm{H}$, Blennow $\mathrm{K}$, et al. Accuracy of brain amyloid detection in clinical practice using cerebrospinal fluid $\beta$-amyloid 42: a cross-validation study against amyloid positron emission tomography. JAMA Neurol 2014;71:1282-9.

4 Olsson B, Lautner R, Andreasson U, et al. CSF and blood biomarkers for the diagnosis of Alzheimer's disease: a systematic review and meta-analysis. Lancet Neurol 2016;15:673-84

5 Sunderland T, Linker G, Mirza N, et al. Decreased beta-amyloid1-42 and increased tau levels in cerebrospinal fluid of patients with Alzheimer disease. JAMA 2003;289:2094-103.

6 Ewers M, Mattsson N, Minthon L, et al. CSF biomarkers for the differential diagnosis of Alzheimer's disease: a large-scale international multicenter study. Alzheimers Dement 2015;11:1306-15.

7 Schoonenboom NSM, Reesink FE, Verwey NA, et al. Cerebrospinal fluid markers for differential dementia diagnosis in a large memory clinic cohort. Neurology 2012;78:47-54 
8 Mattsson N, Insel PS, Donohue M, et al. Predicting reduction of cerebrospinal fluid $\beta$-amyloid 42 in cognitively healthy controls. JAMA Neurol 2015;72:554-60.

9 Sutphen CL, Jasielec MS, Shah AR, et al. Longitudinal cerebrospinal fluid biomarker changes in preclinical Alzheimer disease during middle age. JAMA Neurol 2015;72:1029-42.

10 Blennow K, Dubois B, Fagan AM, et al. Clinical utility of cerebrospinal fluid biomarkers in the diagnosis of early Alzheimer's disease. Alzheimers Dement 2015;11:58-69.

11 Ritchie C, Smailagic N, Noel-Storr AH, et al. Plasma and cerebrospinal fluid amyloid beta for the diagnosis of Alzheimer's disease dementia and other dementias in people with mild cognitive impairment (MCI). Cochrane Database Syst Rev 2014;(6):CD008782

12 Ritchie C, Smailagic N, Noel-Storr AH, et al. CSF tau and the CSF tau/ABeta ratio for the diagnosis of Alzheimer's disease dementia and other dementias in people with mild cognitive impairment (MCI). Cochrane Database Syst Rev 2017;3:CD010803.

13 Hampel H, Toschi N, Baldacci F, et al. Alzheimer's disease biomarker-guided diagnostic workflow using the added value of six combined cerebrospinal fluid candidates: $A \beta_{1}$. ${ }_{42}$ ' total-tau, phosphorylated-tau, NFL, neurogranin, and YKL-40. Alzheimers Dement 2018:14:492-501.

14 O'Bryant SE, Mielke MM, Rissman RA, et al. Blood-Based biomarkers in Alzheimer disease: current state of the science and a novel collaborative paradigm for advancing from discovery to clinic. Alzheimers Dement 2017;13:45-58.

15 Mattsson N, Zetterberg H, Janelidze S, et al. Plasma tau in Alzheimer disease. Neurology 2016;87:1827-35.

16 Nakamura A, Kaneko N, Villemagne VL, et al. High performance plasma amyloid- $\beta$ biomarkers for Alzheimer's disease. Nature 2018;554:249-54.

17 Herring A, Münster Y, Akkaya T, et al. Kallikrein-8 inhibition attenuates Alzheimer's disease pathology in mice. Alzheimers Dement 2016;12:1273-87.

18 Albert MS, DeKosky ST, Dickson D, et al. The diagnosis of mild cognitive impairment due to Alzheimer's disease: recommendations from the National Institute on AgingAlzheimer's association workgroups on diagnostic guidelines for Alzheimer's disease. Alzheimers Dement 2011;7:270-9.

19 McKhann GM, Knopman DS, Chertkow H, et al. The diagnosis of dementia due to Alzheimer's disease: recommendations from the National Institute on Aging-
Alzheimer's association workgroups on diagnostic guidelines for Alzheimer's disease. Alzheimers Dement 2011;7:263-9.

20 Shimizu-Okabe C, Yousef GM, Diamandis EP, et al. Expression of the kallikrein gene family in normal and Alzheimer's disease brain. Neuroreport 2001;12:2747-51.

21 Bao J, Wang X-jie, Mao Z-fu. Associations between genetic variants in 19p13 and $19 q 13$ regions and susceptibility to Alzheimer disease: a meta-analysis. Med Sci Monit 2016;22:234-43

22 Coon KD, Myers AJ, Craig DW, et al. A high-density whole-genome association study reveals that APOE is the major susceptibility gene for sporadic late-onset Alzheimer's disease. J Clin Psychiatry 2007;68:613-8.

23 Nho K, Kim S, Horgousluoglu E, et al. KLK8 as a modulator of Alzheimer's disease pathology: neuroimaging genetics. Alzheimers Dement 2017;13:P966-8.

24 Cissé M, Halabisky B, Harris J, et al. Reversing EphB2 depletion rescues cognitive functions in Alzheimer model. Nature 2011;469:47-52.

25 Mönning U, Sandbrink R, Weidemann A, et al. Extracellular matrix influences the biogenesis of amyloid precursor protein in microglial cells. J Biol Chem 1995;270:7104-10.

$26 \mathrm{Xu} \mathrm{J}$, de Winter F, Farrokhi C, et al. Neuregulin 1 improves cognitive deficits and neuropathology in an Alzheimer's disease model. Sci Rep 2016;6:31692.

27 Djogo N, Jakovcevski I, Müller C, et al. Adhesion molecule L1 binds to amyloid beta and reduces Alzheimer's disease pathology in mice. Neurobiol Dis 2013;56:104-15.

28 Keyvani K, Münster Y, Kurapati NK, et al. Higher levels of kallikrein-8 in female brain may increase the risk for Alzheimer's disease. Brain Pathol 2018;28:947-64.

29 Shaw JLV, Diamandis EP. Distribution of 15 human kallikreins in tissues and biological fluids. Clin Chem 2007;53:1423-32.

30 Molinuevo JL, Ayton S, Batrla R, et al. Current state of Alzheimer's fluid biomarkers. Acta Neuropathol 2018;136:821-53.

31 Henriksen $\mathrm{K}, \mathrm{O}^{\prime} \mathrm{Bry}$ ant $\mathrm{SE}$, Hampel $\mathrm{H}$, et al. The future of blood-based biomarkers for Alzheimer's disease. Alzheimers Dement 2014;10:115-31.

32 O'Bryant SE, Gupta V, Henriksen K, et al. Guidelines for the standardization of preanalytic variables for blood-based biomarker studies in Alzheimer's disease research. Alzheimers Dement 2015;11:549-60.

33 Snyder HM, Carrillo MC, Grodstein F, et al. Developing novel blood-based biomarkers for Alzheimer's disease. Alzheimers Dement 2014;10:109-14. 